\title{
Design and Implementation of Web-based Quality Supervision Administration System of Hydraulic Projects
}

\author{
Ye Jie \\ Guizhou University \\ GuiyangÿChina \\ yejiec@21cn.com
}

\begin{abstract}
In view of the demand of quality supervision information of hydraulic projects of one province, and based on the project quality supervision laws and regulations of China, and the local laws and regulations of the province, the web-based quality supervision administration system of hydraulic projects was designed, which embodies the quality supervision business process of the provincial hydraulic projects. The system adopted SQL Server 2008 database and .NET platform. It consists of function modules of system management, registry and verification management, project startup, inspection and quality assessment, process quality control and test/acceptance. The system covers all the data of the project quality supervision from the subprojects and the quality supervising organizations during the period from the preconstruction of the project to the project acceptance. Furthermore, the system can confine an individual user to a certain operation function according to its role in the project construction. The trial operation of the system shows that it can realize information sharing between multiple responsible entities and project quality supervising organizations during the process of quality supervision and achieve collaboration between them. Therefore it can greatly improve supervision efficiency and reinforce supervision intensity. It has satisfactory practical value.
\end{abstract}

Key words- project quality; supervision; hydraulic; .net platform;SQL Server2008

\section{INTRODUCTION}

"For generations, quality first"! Project quality control is not only the responsibility of the government, but also a matter of people's lives and property safety. To ensure project quality and prevent occurrence of various kinds of illegal practices, a more effective supervision mechanism is needed for the constructor and the supervisor of one project ${ }^{1}$ in addition to the market mechanism and legal binding. To strengthen project quality supervision and improve construction safety supervision of various supervising departments, it is imperative to reform the supervision mechanism.

The quality supervision management of one province's hydraulic projects still belongs to the traditional mode of afterward handling and paper archiving. The project quality supervision adopts traditional sample inspection and interviewing. There are a lot of requirements as to implementation of project construction from departments of discipline inspection and supervision at the state level and the provincial level. But basically they are not able to be fulfilled owing to lack of proper means and tools. During the process of project quality control, there is no information sharing between multiple responsible entities and project supervision organizations and there is no collaboration between them. Thus, supervision efficiency and quality are greatly discounted.

In view of the demand of quality supervision information of hydraulic projects of the province, the quality supervision administration system of hydraulic projects of $\mathrm{B} / \mathrm{S}$ mode was constructed under the environment of Visual Studio.net and SQL SERVER 2008, which makes use of internet/intranet technology, integrates business and supervision to achieve enterprise information sharing and supervision streamlining, graphic digital delivering and integrative documenting so as to adapt to the work requirement of quality and safety supervision management: normalization, institutionalization, standardization, informatization under the new situation. It effectively integrates the management of project quality supervision with information technology to realize collaboration and information sharing between the quality supervision department, the constructor, the supervisor, the employer and the inspection organization so as to provide a real-time monitoring to the supervision work for the supervising organization. In this case the construction situation can be learned timely and the supervision workload and efficiency can be improved and the supervision is under control and monitoring.

\section{REQUIREMENT DESCRIPTION}

The Hydraulic Project Quality Administration Regulation (MWR Order No. 7) issued by Ministry of Water Resources in 1997 clearly specifies: hydraulic projects implement the integrated quality management system that is under the responsibility of the project legal person (employer), under the control of the supervisor, guaranteed by the constructor and supervised by the government. ${ }^{[1]}$

In order to do well in quality supervision of hydraulic project construction and facilitate operation and provide evidence basis during the process of quality supervision, the construction quality supervision mainly consists of pre-construction stage, construction stage and stage of inspection and acceptance. The supervision flow chart is as illustrated in Figure 1: 


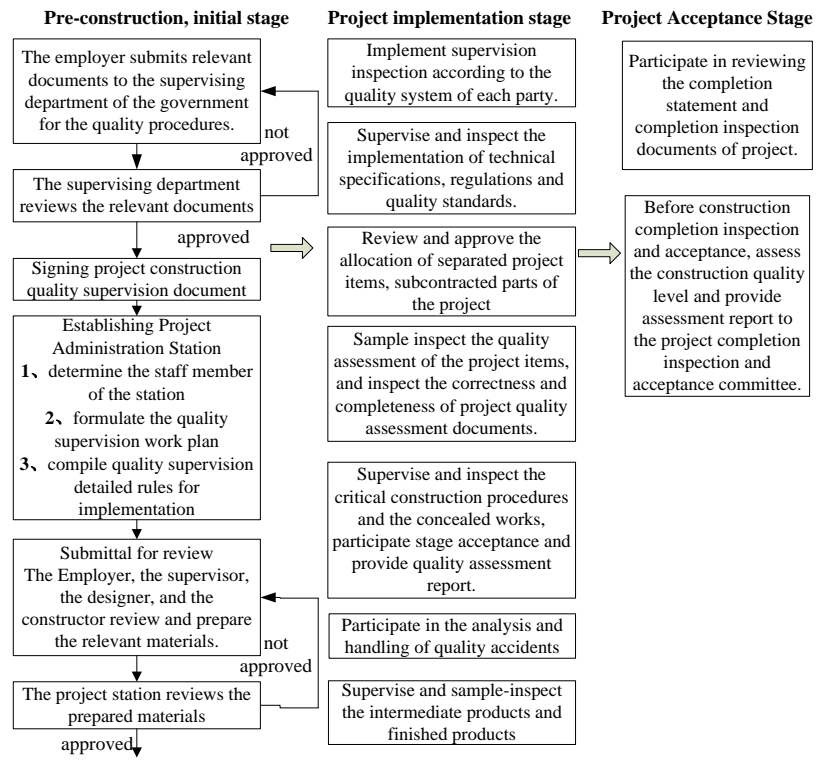

Figure 1. Flow chart of Project Construction Quality Supervision

\section{SYSTEM DESIGN}

The design of the whole system is based on Figure 1: the flow chart of project quality supervision, consisting of three stages: the initial stage of project construction, project construction stage and project acceptance stage, covering all the project-related data of the supervision department, the employer, the supervisor, the designer, the constructor and the inspection and acceptance side involved in the project construction. It also realizes the expanding horizontally and vertically, i.e. the system can accommodate several projects and each project contains all the data of the project quality supervision flow chart.

\section{A. System Architecture}

The system adopts Internet network and browser/Server (B/S) architecture, enabling all-level operators to have an access to the system through ADSL or $3 \mathrm{G}$ network transmission ${ }^{(7)}$, not needing software installation. The network topology of the system is illustrated as Figure 2.

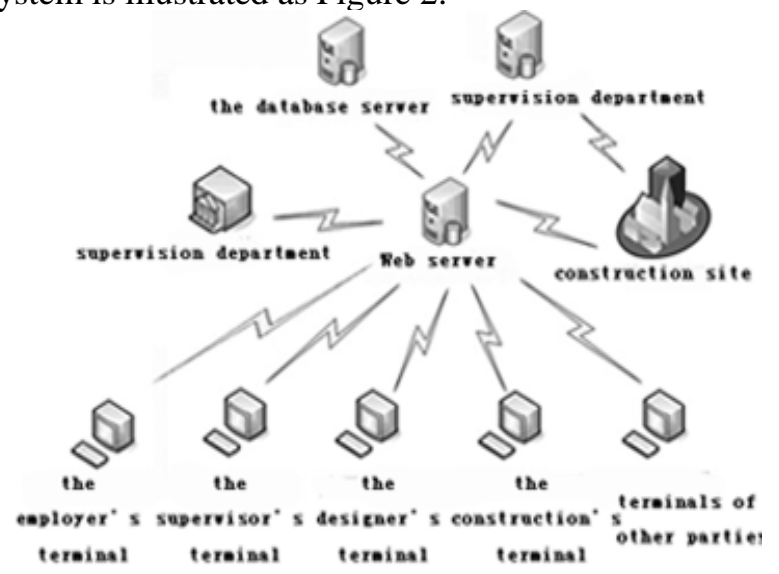

Figure 2. Network Topology of the System
- To establish the unified hydraulic project database server of the province to realize unified data administration of the whole province.

- To establish the unified hydraulic project "WEB server" of the province to realize the access to the system from the supervision department and all parties involved in the project construction.

- To establish digital identity (U certificate) authentication system according to the national requirement. Function 1: to authenticate the operators at all levels and enforce post responsibility system at the same time. Function 2 : to provide encryption for the protected projects.

\section{B. Database Design}

The system involves a great quantity of data and needs to store a great quantity of information. According to the detailed situation and the demand of the system maintenance of the project, Microsoft SQL Server2008 was adopted as the database management system for the system. The system mainly includes the system management information, the information of the employer, the supervisor, the constructor, the designer and inspecting and acceptance institute during the process of project quality supervision management, the project's registry information, project startup information, quality supervision document information, information submitted for approval, project item allocation information involved in the project construction stage, the large quantity of separated item project assessment information, subproject assessment information, unit project assessment information and other items in the quality inspection stage, totally over 80 database tables. The diagram of the systematic partial database tables is illustrated as in Figure 3:

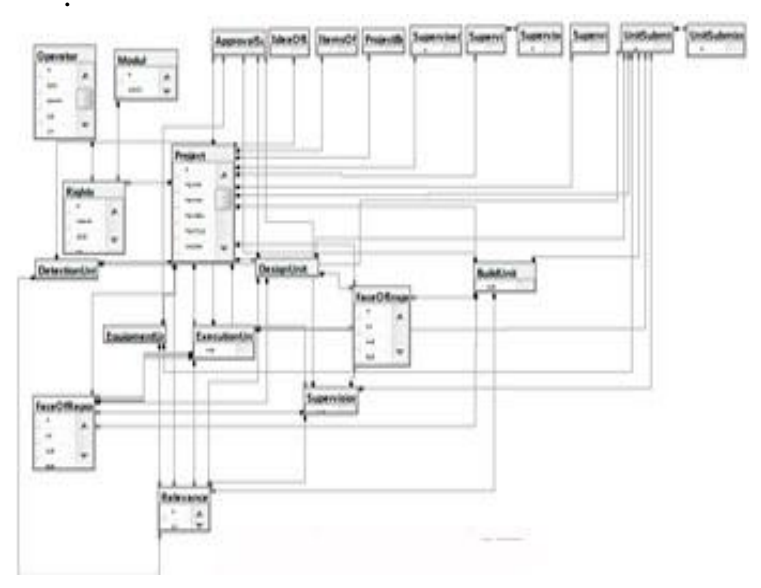

Figure 3. Diagram of Partial Database Tables

\section{System Role}

According to requirements of the system users, there are three kinds of system roles: system administrator, supervisor and constructor. Users in different roles may have different permissions to access the system and the functional interface available is also different. Figure 4 is the diagram of the system roles and interface. 


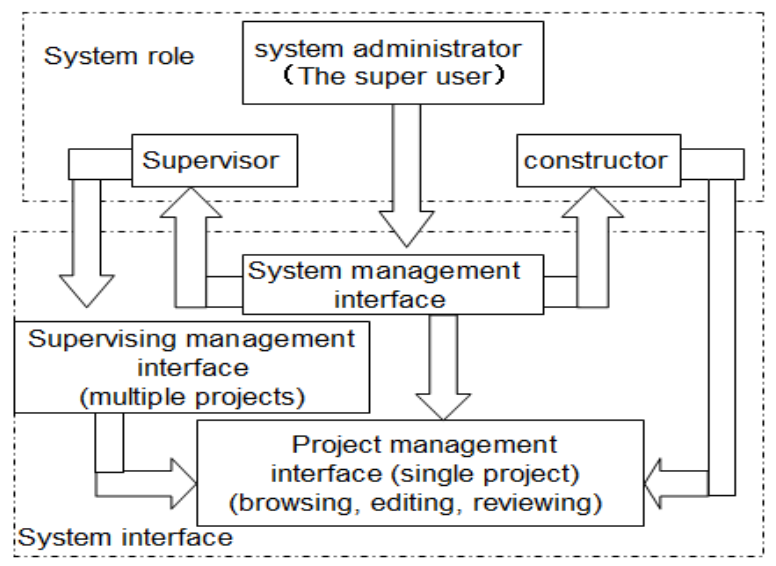

Figure 4. Diagram of the system role and interface

\section{Function Design}

Through actual investigation, and based on national relevant policies, guidelines, laws and regulations and the relevant technical regulations, specifications and quality standards of water conservancy and hydropower industry, and according to the different operation modules and functions permitted to the users, the system functional module with administrator right is presented in Figure 5.

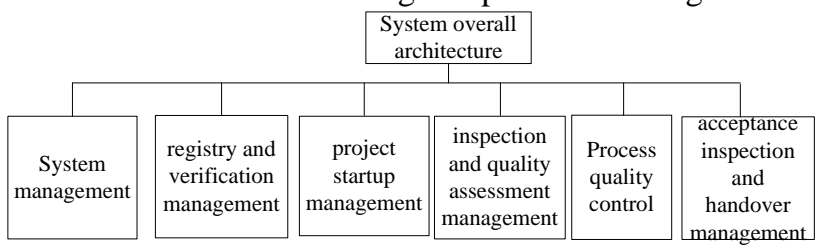

Figure 5. Diagram of system functional module

\section{(1)System management}

It provides setup and management of the whole system for the system administrator. Functions include:

(1) User management

It is used for adding, modifying, deleting and querying user information.

(2) Module management

It is used for adding, modifying, deleting system functional modules. The system functional modules fall into two stages.

\section{(3) Project management}

It is used for adding, modifying, deleting and querying project information.

(4) Permission rights management ${ }^{[6]}$

Allocate different modules for the existing users according to their roles and allocate them different permission rights for different construction projects. When a user logs onto the system, only the operation functional module within his permission rights will be displayed. And the operation rights can be divided into browsing, editing and verification.

(5) Data backup and recovery

It is used for backup and recovery of the system data. It is possible to select all or part of data for backup or recovery.

(6) Log management

It is used for query and deleting of the system logs.
(2)Registry and Verification Management

It provides project reporting and verification before project startup for the constructor and supervisor. Its function includes:

(1) Project registry

The constructor fills in the names of the construction organizations, the general information of the project and the longitude and latitude of the project, and illustrates them in a map.

(2) Quality supervision document

The constructors fill out the quality supervision document according to the standard format. Then the supervising department verifies the document. After being verified, it will enter the module of submittal for approval and provides the export function for the supervision document according to the standard format.

(3) Supervision detailed rules

Fill in the supervision detailed rules in form of WORD and export them.

(4) Management of tables of submittal for approval

The employer, the supervisor, the designer and the constructor fill in the tables of submittal for approval, and then submit them to the supervising department for approval and provide export in the standard format.

(3)Project startup management

To provide the constructor and the supervisor to fill in the startup information and the review work information of the project. Its function includes:

(1) Pre-construction information management

To fill the pre-construction information in WORD format, including mechanical equipment, procurement of equipment and materials, determination of quarry and exploitation information, etc., supporting function of text editing and exporting.

(2) Construction startup information management [2]

To fill in the complete information of the project startup in a standard format and review the materials and provide function of exporting in standard format.

(3) Project information disclosure

To fill in disclosure notice in format of WORD, contents including the responsibilities of various constructors, work content, work means, requirements, etc., supporting function of text editing and exporting.

(4) Project division

To fill in the project division table in standard format, mainly embodied in the division of unit project and division of subproject, and to review them and provide exporting in standard format.

(4) Inspection and quality assessment management

It provides inspection to the separated item project and quality assessment to subproject and unit project during the implementation of construction project. The function includes:

(1) Quality assessment of unit project ${ }^{[3]}$

The inspecting organization or the constructor inspect by themselves, then fill in the unit project quality assessment table with the results by type and review them and it provides function of exporting in standard format.

(2) Quality accident management ${ }^{[3]}$ 
The problematic unit project and work procedure should be recorded in written file after filling in the quality defect with a standard format and be reviewed. It should provide function of exporting in standard format.

(3) Subproject quality assessment ${ }^{[3]}$

After completion of subproject, fill in subproject quality assessment table in standard format and review it, providing function of exporting in standard format.

(4) Unit project quality assessment ${ }^{[3]}$

After completion of unit project, fill in unit project quality assessment table in standard format and review it, providing function of exporting in standard format.

(5) Appearance quality assessment ${ }^{[3]}$

After completion of unit project, according to types of hydraulic structure, building and embankment, fill in hydraulic structure appearance quality assessment table and housing construction and installation appearance quality assessment table in standard format and review them, providing function of exporting in standard format.

(6) Project construction quality assessment $\left.{ }^{[3}\right]$

After completion of the whole project, fill in the project construction quality assessment table in standard format and review it, providing function of exporting in standard format.

(5)Process quality control

It provides inspection and supervision to the constructors during the implementation process of construction project. The function includes:

(1) Supervision and inspection

The supervisor carries out inspection to various constructors and fills the inspection form in standard format according to the difference of organizations.

(2) Quality assessment supervision

The supervisor carries out supervision over the quality assessment and fills in the quality assessment report in standard format.

(3) Progress report management

It refers to the log, weekly report and monthly report of the supervisor and the daily report, weekly report and monthly report of the constructors.

(6) Acceptance Inspection/handover management

(1) Subproject acceptance inspection/handover management

To fill in subproject inspection and acceptance assessment document in standard format and review it, providing function of exporting in standard format.

(2) Unit project acceptance inspection ${ }^{[3]}$

To fill in unit project inspection and acceptance assessment document in standard format and review it , providing function of exporting in standard format.

(3) Contract Project acceptance inspection ${ }^{[3]}$

To fill in contract project inspection and acceptance assessment document in standard format after completion and review it, providing function of exporting in standard format.

(4) Stage acceptance ${ }^{[3]}$

To fill in stage inspection and acceptance assessment document in standard format and review it, providing function of exporting in standard format.

(5) Generator set startup testing and acceptance [3]
To fill in generator set startup inspection and acceptance assessment document in standard format and review it, providing function of exporting in standard format.

6) Acceptance inspection for service of project part [3]

To fill in inspection and acceptance assessment document for service of project part, and review it, and provide function of exporting in standard format.

\section{THE RELEVANT TECHNICAL DETAILS IN THE SYSTEM DEVELOPMENT}

The system used $\mathrm{B} / \mathrm{S}$ structure, and in the environment of Visual Studio 2010, combined with technologies of SQL SERVER 2008 Database Management System, JavaScript and JQuery Controls as well as DevExpress Plug-in, etc. The problems encountered in the development process can be solved. As too many problems have been encountered in the development process, only the following two characteristics are described.

\section{A. Implementation of user rights control}

The system involves setting permission rights to different modules for different users. The permission rights include browsing, editing and auditing and it is stipulated that the auditing permission mush be accompanied by the browsing permission. From a perspective of technology implementation, the users display is realized by Gridview Controls. Because the module is of 2-stage structure, the user permission display is implemented by using TreeView Controls, and the permission control is realized by combining client-side coding with server-side coding. The following is the main code of the permission display and control.

The server-side 2-stage module and the permission display:

string strSql = "SELECT id,M_name from Modul where up_id=0 "; //remove the top-level module from the module table and put it into the data set and traverses each node in turn to establish the parent node of TreeView.

foreach (DataRow dr in dt.Rows) //traversing the root node

$\{\quad \ldots . . . \ldots . .$. the root node

TreeView1.Nodes.Add(Root); //establishing string $\operatorname{str}=$ "SELECT $*$ from Rights,Modul where Modul.id=Rights.ModulID and odul.up_id $="+$ dr["id"].ToString() + " and UserID=" + uid+" and $\mathrm{PID}="+\mathrm{pid}$;// remove the sub-module of various toplevel modules from the module table and traverses the sub-module and remove the module name and permission.

\section{DataSet dsNode $=$ new $\operatorname{DataSet}()$}


dsNode

$$
=
$$

bc.GetDataSet(str,

"tempNode");//remove the corresponding permission of the user from the access matrix and place it in sNode

Nodeselect. Text $=$ rootss;

Root.ChildNodes.Add(Nodeselect);

foreach (DataRow drNode in dtNode.Rows) //establishing root node traversing

\{

TreeNode Node $=$ new TreeNode () ;

int see =

Convert.ToInt16(drNode["RightString"].ToString().Su

bstring $(0,1))$;//remove browsing permission

if $($ see $==0)$

ISsee = "false"; //set permissions based on

the removed value,

else

ISsee = "true";

string var $=$ drNode["id"].ToString ()$\cdot$ Trim () ;

string seename $=$ "see" +

dr["id"].ToString()+drNode["id"].ToString();

//browsing permission various controls display, adopting client-side controls input type $=$ 'checkbox', adopting JavaScript to write code to control Controls class='see' string ssee = clinput type $=$ 'checkbox' dr["id"].ToString()+drNode["id"].ToString() + ")' id="' + seename + "' name='see' value="' + var + "'> <script language $=$ javascript $>$ " + seename + ".checked $="+$ ISsee $\quad+" ;<$ script $><\mathrm{a} \quad$ onclick='seecheckbox(" + dr["id"].ToString()+drNode["id"].ToString() + ")' class='seetext' >browsing permissionT $</ \mathrm{a}>$ ";

String ss $=$ name + nul + ssee + sedit + saudi + sradio1 + sradio2; //sub-module name and various permissions display

Node. Text $=\mathrm{ss}$;

Root.ChildNodes.Add(Node); // add submodule in treeview

Get the user's permission selection results and store them in the data table in form of unicode string

if (Request.Form["see"] != null) //get browsing permission, what has been stored in see is the id string all selected

\{

string see $=$ Request.Form["see"].ToString(); if (see != "")

\{

string[] see_str = see.Split(','); //split the

string and remove the id from every selected module int $\mathrm{i}$;

for (i $=0 ;$ i $<$ see_str.Length; i++)<smiles>[Tl]</smiles>

if(see_str[i].ToString()

dtr["id"].ToString()) //if moduleID is in the selected string ssee $=" 1 " ; \quad / /$ set the browsing permission of the module to be the selected state

\}

string rightstring $=$ ssee + sedit + saudi;//split joint the browsing permission, editing permission and auditing permission and store them in the database table

sqlstr = "update Rights set RightString="" + rightstring + "'where id=" + dtr["id"].ToString();

bool ent $=$ bc.UpSQL( sqlstr);

//use client-side JavaScript control permission setting control to set the code of checkbox

function seecheckbox(id) \{

var cn $1=$ "see" + id; $\quad$ var cn $2=$ "edit" + id; var cn3 = "audi" + id;

document.getElementById(cn1).checked

$=$ !document.getElementById(cn1).checked;//set the browsing permission selected or not false) \{ if (document.getElementById(cn1).checked ==

document.getElementById $(\mathrm{cn} 2) \cdot$.checked = false; //the browsing permission not selected, the editing permission also not selected

document.getElementById(cn3).checked = false; // the browsing permission not selected, the auditing permission also not selected

\section{B. Implementation of content dynamic change and operation in the nested table}

The data table submitted in hydraulic projection supervision application involves many items of information related to names, picture list, document copies number, notes, etc. Each item includes several lines and each line of the picture list has more than one line. Each line corresponds to one information, which can be uploaded and deleted separately. The uploading of information for each line is controlled by a unified control while the deletion can be controlled independently. In view of this complicated situation, especially tables set in tables, we used GridView nested with GridView to implement this, and realize the content control and dynamic operation by writing codes. The main codes are as follows:

// save data into the data table of SuperviseDatasImg in the upload button

((FileUpload)GridView1.Rows[row].FindControl( "FileUpload3")).SaveAs(path); //the server saving path

bool flag = bc.InsertImg2Db (path, "SuperviseDatasImg", id );//picture binary streams saved into the database

// writing code to bind the data nested in 
GridView for RowDataBound incident nested in GridView.

GridView

Grd

$=$

(GridView)e.Row.Cells[2].FindControl("GridView2");

//finding Control, GridView2 is the nested table

string

id

GridView1.DataKeys[e.Row.RowIndex].Value.ToStrin

$\mathrm{g}()$;

string sqlstr $=$ "select $*$ from SuperviseDatasImg

where $\operatorname{sid}="+\mathrm{id}$;

DataSet dss = new DataSet () ;

dss = bc.GetDataSet(sqlstr, "'"); //remove the data of each line from the data table and put them into the dataset dss

DataTable temp = new DataTable("tab");

temp.Columns.Add("name",

System.Type.GetType("System.String"));

//add

information name in the data source.

temp.Columns.Add("id",

System.Type.GetType("System.String")); //add the id number of information in the data source

DataRow[] rows $=$ dss. Tables[0].Select () ;

if (rows.Count ()$!=0)$

$\{$ int $\mathrm{i}=0$;

foreach (DataRow row in rows) //put the data into the corresponding line of the related data table

\{ i++;

DataRow newrow = temp.NewRow () ; newrow $[0]=$ "information" + i.ToString () ;

newrow[1] = row["id"];

temp.Rows.Add(newrow);

\} \}

Grd.DataSource = temp;

Grd.DataBind(); // GridView2 data binding

writing the following codes in the deletion button :

String

$\mathrm{id}=$

((ImageButton)sender).CommandArgument.ToString()

;//series number of pictures

string sqlstr $=$ "select sid from SuperviseDatasImg where $\mathrm{id}=" \mathrm{id}$;

string $\operatorname{sid}=$ bc.getvalue(sqlstr); //serial number of information

string delstq $=$ "delete from SuperviseDatasImg where $\mathrm{id}="$ + $\mathrm{id}$; //delete the corresponding information of the data table

bc.UpSQL(delstq);

sqlstr = "select Sup_Data_Count from
SuperviseDatas where id=" + sid;

int count

Convert.ToInt16(bc.getvalue(sqlstr));

count--;

sqlstr = "update SuperviseDatas set

Sup_Data_Count=" + count + " where id=" + sid;

bc.UpSQL(sqlstr); // modify the number of information in the corresponding line

\section{CONCLUSION}

By establishing project quality supervision system, based on the traditional manual management mode, database and network business process management were integrated to make project quality supervision more normalized, standardized and highly efficient. Currently the system is under trial operation. The system operation is in good condition, it can fully reflect the process of project quality supervision, complying with the actual needs of the hydraulic project quality supervision of the province. Besides, with a friendly interface, it is easy to operate, and it can basically meet the demands of the supervising organization and the other parties involved in the project construction. For the next step, we will develop the decision-making support system based on data mining on the basis of this system to meet further needs of users.

\section{REFERENCES}

[1] Code of Hydraulic Project Construction Quality Inspection and Assessment, Ministry of Water Resources of P.R.China (SL1762007)(M), Beijing: China Water and Power Press, 2007

[2] Hydraulic Project Construction Supervision Specification of P.R.China(SL288-2003)[M], Beijing: China Water and Power Press, 2003

[3] Hydraulic Project Construction Quality Evaluation Form New Specified Form, Ministry of Water Resources of P.R.China[M], Beijing: China Water and Power Press, 2003

[4] Desmond Francis D'souza. Objects Componentw and Frameworks with UML[M].pearson Education 2003

[5] Ding Shifeng,Zhu Yi, Yang Mingyu. Proficiency in C\#3.0 and NET 3.5 Advanced Programming: LINQ,WCF,WPF,WF[M] Beijing: Tsinghua University Press, 2009

[6] Liu Jinxiao. Research and Implementation of Permission Control in Web Application System[J],Computer Engineering \& Design 2008,29(10):2550-2553

[7] Chen Ren, Tan Hui, Tong Yin, Chen Li. Enterprise Group Comprehensive Running Management Based on Web Service[J], Computer Application and Software, 2019, 29(1):203-204

[8] Cao Xiangzhi. Software Testing Program Practice-Technology, Process and Management, Beijing: Publishing House of Electronics Industry, 2009

[9] Atenies G, Pietro R D, Mancini L V, Tsudik G..Scalable and Efficient Provabel Data Possession. In Proc. of SecureComm 2008. $1 \sim 10$

[10] Dong mei Chen, zheng hua hu. User oriented information system interface design and implementation method research [J]. Journal of modern information technology, 2007 (11); 49-53 